\title{
Discrimination of muons for mass composition studies of inclined air showers detected with IceTop
}

\section{The IceCube Collaboration}

(a complete list of authors can be found at the end of the proceedings)

E-mail: aswathi.balagopalv@icecube.wisc.edu

IceTop, the surface array of IceCube, measures air showers from cosmic rays within the energy range of $1 \mathrm{PeV}$ to a few $\mathrm{EeV}$ and a zenith angle range of up to $\approx 36^{\circ}$. This detector array can also measure air showers arriving at larger zenith angles at energies above $20 \mathrm{PeV}$. Air showers from lighter primaries arriving at the array will produce fewer muons when compared to heavier cosmic-ray primaries. A discrimination of these muons from the electromagnetic component in the shower can therefore allow a measurement of the primary mass. A study to discriminate muons using Monte-Carlo air showers of energies 20-100 PeV and within the zenith angular range of $45^{\circ}-60^{\circ}$ will be presented. The discrimination is done using charge and time-based cuts which allows us to select muon-like signals in each shower. The methodology of this analysis, which aims at categorizing the measured air showers as light or heavy on an event-by-event basis, will be discussed.

Corresponding authors: Aswathi Balagopal V. ${ }^{1 *}$

${ }^{1}$ Wisconsin IceCube Particle Astrophysics Center, University of Wisconsin, Madison, WI 53703, USA

* Presenter 


\section{Introduction}

The determination of the primary mass composition of cosmic rays with varying energies provides added information about the origin of the cosmic rays that contribute to the observed cosmic-ray spectrum and their propagation within the Universe. A common method to determine the mass of the cosmic-ray primary is with the use of the muon content within the air shower. Heavy primaries, like iron, will generate a larger number of muons in the air shower than light primaries, like proton, of the same energy.

In this analysis, we explore a method that can be used to determine the light or heavy mass group of the primary cosmic ray on an event-by-event basis. The study is performed for inclined air showers detected by the IceTop array. Such a study is important for the test of existing hadronic interaction models, and the muon content for different mass groups predicted by such models. The composition study for inclined air showers measured by IceTop will also provide an independent cross-check to other composition analyses of IceTop using quasi-vertical air showers. IceTop is the surface component of IceCube, the neutrino telescope at the South Pole. IceTop consists of 162 ice-Cherenkov tanks that measure signals deposited by charged particles arising from cosmic-ray

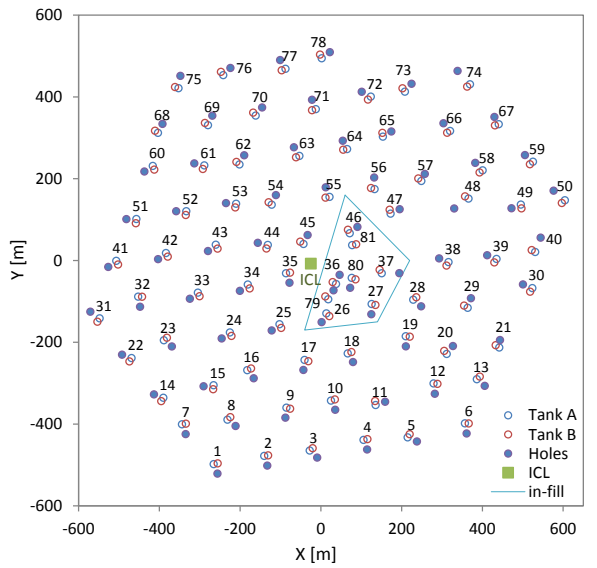

Figure 1: The layout of the IceTop array from [1].

air showers [1]. These 162 tanks are grouped into 81 stations, with each station composed of two tanks. Figure 1 shows the geometry and layout of the IceTop array. Each IceTop tank contains two digital optical modules (DOMs) that collect the deposited Cherenkov light. Since Cherenkov light is emitted by both muons and electromagnetic particles (with non-negligible hadronic contribution), a combination of signals from these will be observed in the tank signal.

There exist several analyses using IceTop and IceCube with the goal of discriminating the electromagnetic particles from the muons. For analyses focused on the determination of the muon density, it is crucial to get a near-perfect number of the observed muons [2]. However, in the cases where the discrimination is used to determine the mass-composition, a contamination of electromagnetic components within the muon parameter can be tolerated [3].

Other analyses using IceTop and IceCube for mass-composition studies operate only for showers with zenith angles up to $36^{\circ}$ [3-6]. Air showers with zenith angles $(\theta)$ larger than this are generally not included within the standard IceTop analyses. This is primarily due to the unavailability of energy calibration (for the energy proxy $S_{125}$ which is the signal expected to be deposited in a tank at a distance of $125 \mathrm{~m}$ ) using simulations for these air showers. However, we can determine the energy of these showers with larger $\theta$ using the method of constant intensities, where we compare the inclined showers to the vertical showers (with the expectation of universal flux rates) to determine their energy [7]. Therefore, we can use such inclined air showers also for mass-composition studies; which is done in this analysis. 


\section{Analysis method}

We use air-shower simulations that were generated using CORSIKA [8], with Sibyll 2.1 [9] as the hadronic-interaction model. We consider simulations with zenith angles above $45^{\circ}$ and energies above $10^{7.3} \mathrm{GeV}$. This is the energy threshold of inclined air showers measured with IceTop [7]. Since the available simulation set (with full detector response) ends at an energy of $10^{8} \mathrm{GeV}$, this is the maximum energy that we consider in this analysis. We divide the showers into three zenith bins: $45^{\circ}-50^{\circ}, 50^{\circ}-55^{\circ}$, and $55^{\circ}-60^{\circ}$. These showers are also divided into different bins of their energy proxy $\log _{10}\left(\mathrm{~S}_{125} / \mathrm{VEM}\right)$, where VEM is the expected amount of charge deposited in a tank by a vertical-equivalent muon. We choose only the bins of $\log _{10}\left(\mathrm{~S}_{125} / \mathrm{VEM}\right)$ where the energy is above $10^{7.3} \mathrm{GeV}$. Also, some energy-proxy bins are dropped due to insufficient statistics for the simulations. The energy-proxy bins that we use are: $\log _{10}\left(\mathrm{~S}_{125} / \mathrm{VEM}\right)=0.7-1.0,1.0-1.3,1.3-1.6$ for $45^{\circ}-50^{\circ}$ showers, and $\log _{10}\left(\mathrm{~S}_{125} / \mathrm{VEM}\right)=0.4-0.7,0.7-1.0,1.0-1.3$ for showers with zenith angles $50^{\circ}-55^{\circ}$ and $55^{\circ}-60^{\circ}[7]$.

We apply cuts to the simulated events to ensure good reconstruction quality for the events used in the analysis. These event-level quality cuts are as follows: 1 . We require 5 IceTop stations to be triggered and passed through the filter. 2. The reconstruction of the air shower is required to have succeeded. 3. The number of layers of tanks around the shower core should be greater than one. This ensures that at least two outer-layers of tanks are there surrounding the shower core. 4. The tank with the maximum signal within the shower should not be located at the edge of the array. Even if the previous cut is applied, we sometimes are left with events where the maximum signal is in the edge, due to noisy hits. Application of this cut ensures better quality for the events chosen. Although we reject $\approx 80 \%$ events with these cuts, the remaining events have good quality, which is needed to conduct this study.

Apart from these event-level quality cuts, we apply some analysis-level cuts to select the muonlike signals in the air showers. The first cut is a charge-based cut. We choose those hits in the tanks which have a charge between 1 and 4 VEM. This was chosen since muons from inclined air showers were mainly seen to fall within this charge range for IceTop. This cut already removes a majority of the electromagnetic contamination within the selected hits; especially at larger distances from the shower core. In addition to this, we apply a time-based cut to the remaining hits in the event. Only those events with $\Delta(\delta \mathrm{t})>0$ are chosen in the final level. Here, $\delta \mathrm{t}$ is the curvature of the shower front. $\Delta(\delta \mathrm{t})=\delta \mathrm{t}_{\text {observed }}-\delta \mathrm{t}_{\text {predicted }}$ compares the observed curvature to the theoretical prediction of the shower curvature (which is optimised for quasi-vertical showers). The condition $\Delta(\delta \mathrm{t})>0$ chooses the hits that arrived before their predicted times. Combined with the charge-based cut, this allows us to choose the early muons in the shower. The addition of this cut further removes electromagnetic contamination from the selected hits. Figure 2 shows the effect of the analysis-level cuts that selects muon-like hits for proton and iron showers.

Once the final-level hits have been selected, they are binned with respect to their perpendicular distance to the shower axis. The sum of all hits above each distance bin is evaluated to obtain the muon-like parameter.

$$
\xi_{\mu-\mathrm{like}}=\Sigma N_{\mu-\mathrm{like}}>r
$$

The lateral distribution of the muon-like parameter, $\xi_{\mu \text {-like }}$ for air showers within the zenith angular range of $55^{\circ}-60^{\circ}$ for the energy bin $\log _{10}\left(\mathrm{~S}_{125} / \mathrm{VEM}\right)=0.7-1.0$ is shown in Figure 3 . 

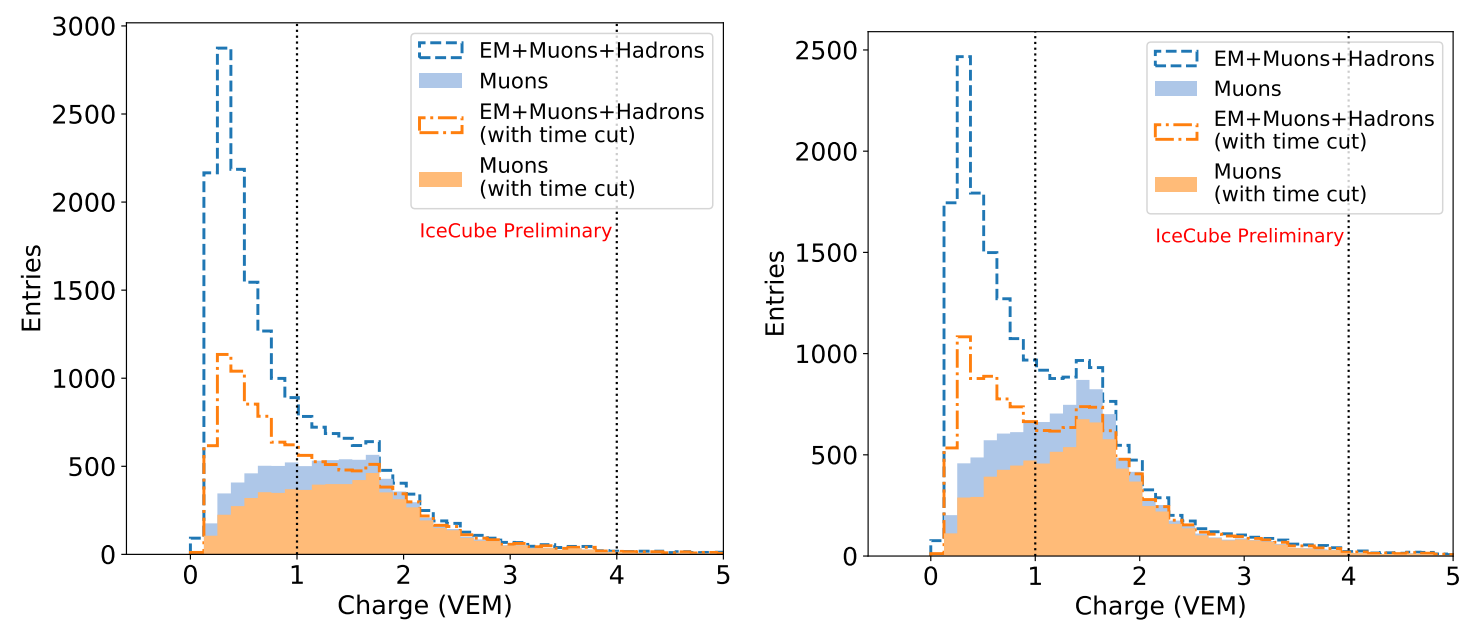

Figure 2: Left: Proton showers, $\theta=50^{\circ}-55^{\circ}$ and $\log _{10}\left(\mathrm{~S}_{125} / \mathrm{VEM}\right)=0.7-1.0$. The charge within the tanks from all particle types for hits with distance from the shower axis $\geq 260 \mathrm{~m}$ are shown. These are the events that pass the event-level quality cuts. The muon-component of these hits are also shown. The charge distribution once the time-based cut is applied is also shown (including its muon content). All hits that fall within the two vertical lines between 1 and 4 VEM will be chosen as the final muon-like hits. Right: The same for iron showers, $\theta=45^{\circ}-50^{\circ}$ and $\log _{10}\left(\mathrm{~S}_{125} / \mathrm{VEM}\right)=1.0-1.3$. The hits with distance from the shower axis $\geq 330 \mathrm{~m}$ are shown.

This is shown for simulated air showers of different primaries. As expected, the mean of $\xi_{\mu-\text { like }}$ exhibits the trend where heavier primaries have more muon-like hits than lighter primaries. We can also see that there is a good amount of separation between the distribution of the light and the heavy primaries. We can therefore use this parameter to determine the lightness/heaviness of each shower. The bottom panel of Figure 3 depicts the fraction of electromagnetic contamination within $\xi_{\mu \text {-like }}$ at each distance bin. The electromagnetic contamination is seen to be small, especially at distances further from the center of the shower.

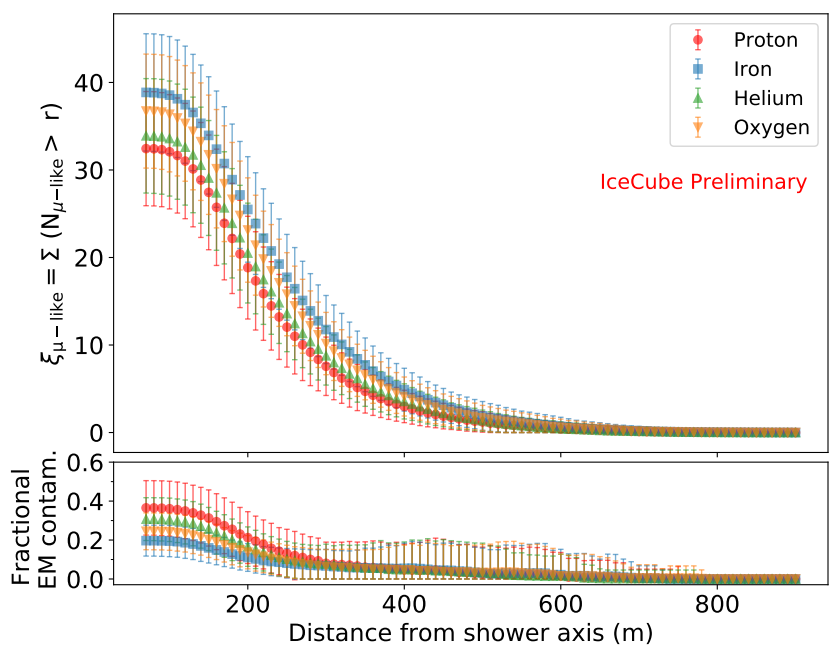

Figure 3: Top panel: lateral distribution of the muon-like

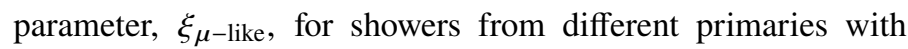
$\theta=55^{\circ}-60^{\circ}$ and $\log _{10}\left(\mathrm{~S}_{125} / \mathrm{VEM}\right)=0.7-1.0$. Bottom panel: corresponding fraction of electromagnetic contamination within signals considered in the $\xi_{\mu \text {-like }}$ calculation. Both the top and bottom panels show the mean values (marker) and standard deviations (error bar) of the respective distribution.

The next step in the analysis is the calculation of the reference distance at which we get the maximum separation between the $\xi_{\mu \text {-like }}$ of the heavy and light primaries. For this we look at the proton and iron primaries alone. A Gaussian distribution is fit to the $\xi_{\mu-\text { like }}$ at each distance bin for both the proton and iron showers. A measure of the separation of these two distributions is estimated to get the distance at which the separation is maximum, with least amount of fluctuations. 
For this, the figure of merit (FOM) is calculated as

$$
\mathrm{FOM}=\frac{|\mu(\mathrm{H})-\mu(\mathrm{Fe})|}{\sqrt{\sigma_{\mathrm{H}}^{2}+\sigma_{\mathrm{Fe}}^{2}}},
$$

where $\mu$ is the mean of the Gaussian distribution and $\sigma$ is the standard deviation [10].

An example of the FOM calculation is given in Figure 4 (left). The figure shows the distributions of $\xi_{\mu \text {-like }}$ at a distance of $260 \mathrm{~m}$ for proton and iron showers with $\theta=55^{\circ}-60^{\circ}$ and with $\log _{10}\left(\mathrm{~S}_{125} / \mathrm{VEM}\right)=0.7-1.0$. The resulting value of the FOM for this distribution is $1.08 \pm$ 0.08. Once the FOM is calculated for each distance bin, they can be compared as shown in Figure 4 (right). The figure shows the FOM values at different distances for $\theta=50^{\circ}-55^{\circ}$ and $\log _{10}\left(\mathrm{~S}_{125} / \mathrm{VEM}\right)=1.0-1.3$. Based on this, the distance of $260 \mathrm{~m}$ is chosen as the reference distance for this bin. This procedure is repeated for all zenith bins and all energy bins used in the analysis.
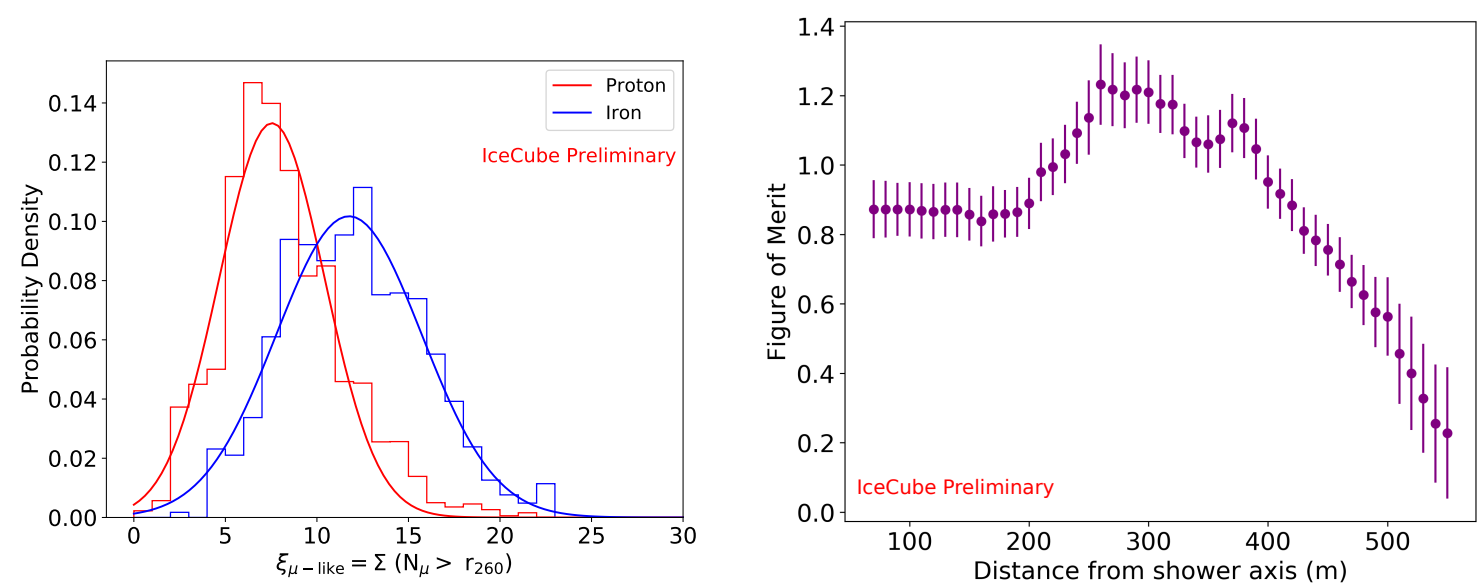

Figure 4: Left: An example of the FOM calculation for showers with $\theta=55^{\circ}-60^{\circ}$ and $\log _{10}\left(\mathrm{~S}_{125} / \mathrm{VEM}\right)=$ $0.7-1.0$. A Gaussian distribution is fit to both iron and proton distributions of $\xi_{\mu-\text { like }}$ for a given distance bin (here $260 \mathrm{~m}$ ). Right: The obtained FOM vs distance for showers with $\theta=50^{\circ}-55^{\circ}$ and $\log _{10}\left(\mathrm{~S}_{125} / \mathrm{VEM}\right)=$ $1.0-1.3$. The reference distance is chosen from this.

Upon the determination of the reference distance, the probability-density distribution for $\xi_{\mu \text {-like }}$ can be drawn for both proton and iron showers. This is done for each zenith and energy bin considered in the analysis. An example of such a distribution for showers with $\theta=50^{\circ}-55^{\circ}$ and $\log _{10}\left(\mathrm{~S}_{125} / \mathrm{VEM}\right)=0.7-1.0$ is shown in Figure 5. The figure shows the two-dimensional probability-density distribution of $\xi_{\mu \text {-like }}$ at the reference distance of $260 \mathrm{~m}$ with respect to $\log _{10}\left(\mathrm{~S}_{125} / \mathrm{VEM}\right)$. The solid-blue curves in the figure represent the $1 \sigma, 2 \sigma$ and $3 \sigma$ contours for the iron showers while the red-dashed curves represent the same for the proton showers. The right panel in the figure also shows the combined $\xi_{\mu \text {-like }}$ distributions of proton and iron showers within the entire bin. A separation between the iron and proton showers is visible, with some amount of overlap. This signifies that in the regions with less overlap, we can identify the showers more confidently, while in the overlap regions the shower primary becomes more ambiguous.

The nature of the iron and proton probability-density distributions is utilised to evaluate the light or heavy nature of each observed air-shower event. For this, the marginal cumulative 


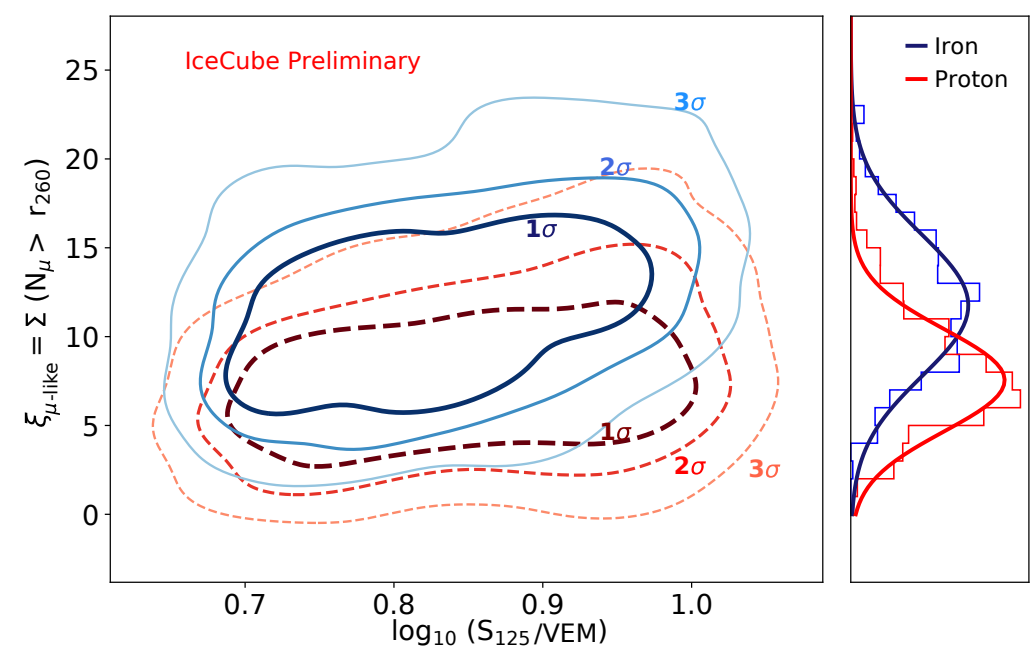

Figure 5: Probability-density distributions for proton (red dashed) and iron (blue solid) showers with $\theta=50^{\circ}-55^{\circ}$ and $\log _{10}\left(\mathrm{~S}_{125} / \mathrm{VEM}\right)=0.7-1.0$ for the reference distance of $260 \mathrm{~m}$. The right panel shows the combined distribution of $\xi_{\mu-\text { like. }}$.

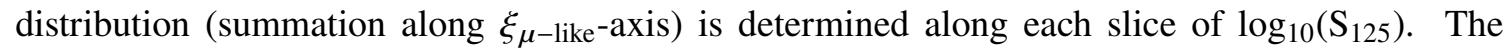
summation is performed along the positive $y$-axis for iron and along the negative $y$-axis for the proton distribution. This results in an iron-marginal cumulative distribution (Fe-CD) value and proton-marginal cumulative distribution (H-CD) value for each point in this space. With this, we can assign a Fe-CD and $\mathrm{H}-\mathrm{CD}$ value to each event with a given pair of $\log _{10}\left(\mathrm{~S}_{125}\right)$ and $\xi_{\mu-\text { like. Any }}$ event with a high H-CD value and low Fe-CD value would be identified as light (or proton-like) and vise-versa for heavy (or iron-like) events.

\section{Testing the procedure}

In order to verify the performance of the analysis procedure, we conduct a test using simulations. The simulated air showers are passed through the analysis to see the fraction of proton/iron events correctly identified as light/heavy.

Figure 6 shows the verification done for iron (left) and proton (right) simulations, on an eventby-event basis. Each simulated event is assigned an H-CD and Fe-CD value, and a histogram of these assigned values is shown in the figure. The colour scale depicts the fraction of events entering each bin in the histogram. Events with low Fe-CD values are considered as light and events with low $\mathrm{H}-\mathrm{CD}$ values are considered as heavy.

We can sum up the fractions in the bottom-first row of bins on the x-axis (with low H-CD value) to determine the percentage of entries identified as heavy. The same can be done for each set of rows. Similarly, the columns can be added up to see the percentage of entries in each column, the leftmost column (with low Fe-CD) being the events most confidently identified as light.

Table 1 shows the percentage of events that enter the bottom-first row (with low $\mathrm{H}-\mathrm{CD}$ value) and the bottom-second row, that are identified as heavy for showers with $\theta=50-55^{\circ}$ and $\log _{10}\left(\mathrm{~S}_{125} / \mathrm{VEM}\right)=0.7-1.0$ (middle $\theta$ and energy bins). The table also shows the percentage of 

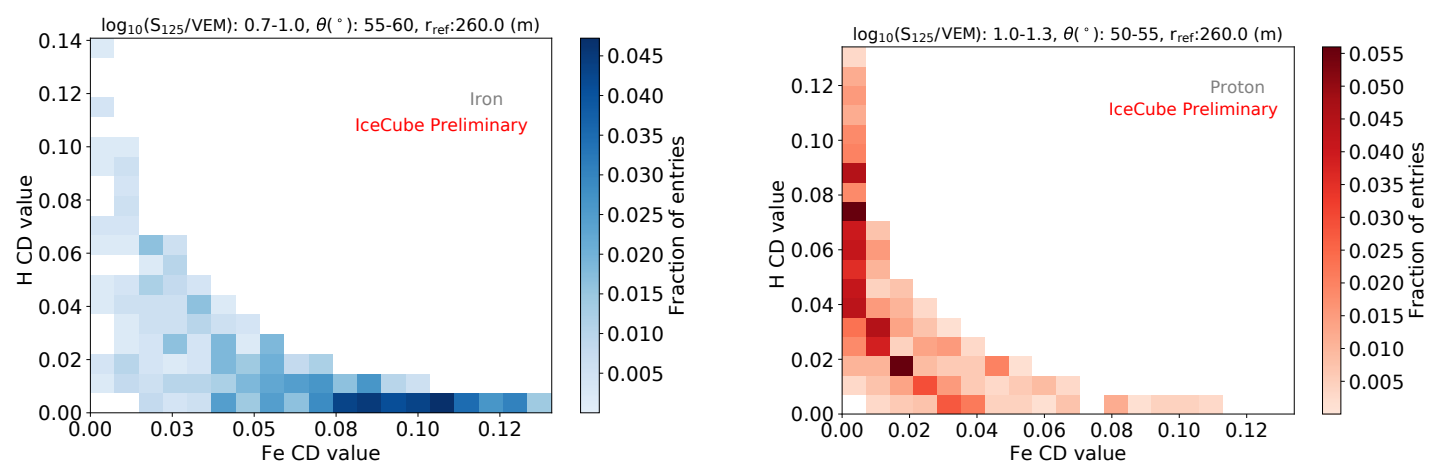

Figure 6: Verification of the mass-classification procedure using simulations. The $\mathrm{H}-\mathrm{CD}$ value and $\mathrm{Fe}-$ $\mathrm{CD}$ value is created for each event and compared. Left: Iron showers $\left(\log _{10}\left(\mathrm{~S}_{125} / \mathrm{VEM}\right)=0.7-1.0\right.$ and $\theta=55-60^{\circ}$ ), all events falling in the bins with low $\mathrm{H}-\mathrm{CD}$ values are correctly identified as heavy. $45.4 \%$ events fall in the 1 st row (correctly identified) and $2.7 \%$ events fall in the 1 st column (misidentified as light). Right: Proton showers $\left(\log _{10}\left(\mathrm{~S}_{125} / \mathrm{VEM}\right)=1.0-1.3\right.$ and $\left.\theta=50-55^{\circ}\right)$, with events falling in the bins with low Fe-CD values identified as light. $46.2 \%$ events fall in the 1 st row (correctly identified) and $12.9 \%$ events fall in the 1 st column (misidentified as heavy)

\begin{tabular}{|c|c|c|c|c|}
\hline Element & $\begin{array}{c}\text { Events in } \\
\text { 1st column } \\
\text { (light) }\end{array}$ & $\begin{array}{c}\text { Events in } \\
\text { 2nd column } \\
\text { (light) }\end{array}$ & $\begin{array}{c}\text { Events in } \\
\text { 1st row } \\
\text { (heavy) }\end{array}$ & $\begin{array}{c}\text { Events in } \\
\text { 2nd row } \\
\text { (heavy) }\end{array}$ \\
\hline True $\mathrm{H}$ & $26.7 \%$ & $14.6 \%$ & $6.2 \%$ & $7.1 \%$ \\
\hline True Fe & $3.3 \%$ & $4.4 \%$ & $40.2 \%$ & $17.1 \%$ \\
\hline True He & $14.7 \%$ & $10.6 \%$ & $16.1 \%$ & $11.6 \%$ \\
\hline True O & $7.1 \%$ & $6.2 \%$ & $21.0 \%$ & $13.6 \%$ \\
\hline
\end{tabular}

Table 1: Testing the classifier for various elements for events that fall in the middle $\theta$ and energy proxy bins $\theta=50-55^{\circ}$ and $\log _{10}\left(\mathrm{~S}_{125} / \mathrm{VEM}\right)=1.0-1.3$.

events in the leftmost column (with low Fe-CD value) and the second column from the left, that are identified as light. The same calculation can be performed for the events shown in Figure 6 also.

It is clear that $40-50 \%$ of iron events are correctly identified as heavy (with better confidence) and $30-40 \%$ of proton events are correctly identified as light. A smaller percentage of proton/iron events are incorrectly identified as heavy/light. One caveat to this is that the same events used for drawing the probability-density distributions have been used to test the procedure, due to the lack of sufficient simulations.

The same procedure can be repeated for the available helium and oxygen simulations to test the classifier. This is shown in Figure 7 for helium and oxygen events with $\theta=45^{\circ}-50^{\circ}$ and $\log _{10}\left(\mathrm{~S}_{125} / \mathrm{VEM}\right)=0.7-1.0$. The helium and oxygen events are not seen to clump as closely to the light/heavy composition bins as the proton/iron events. They show mixed nature, as expected. This shows that the classification is indeed composition sensitive since helium and oxygen should fall as intermediaries. The percentage of helium and oxygen events that fall in the light-identified columns and heavy-identified rows for air showers falling in the middle $\theta$ and $\mathrm{S}_{125}$ bins are shown in Table 1. 

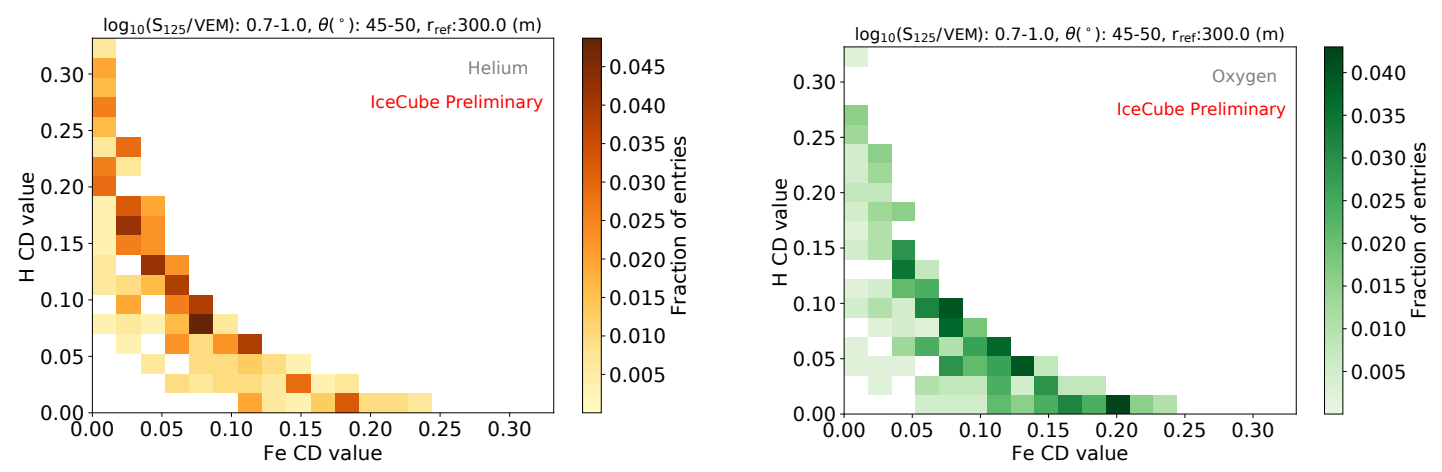

Figure 7: The verification done for helium showers (left) and oxygen showers (right) with, $\left.\log _{10} \mathrm{~S}_{125} / \mathrm{VEM}\right)=0.7-1.0$ and $\theta=45-50^{\circ}$, using the $\mathrm{H}-\mathrm{CD}$ and Fe-CD values obtained from the probability-density distributions obtained from proton and iron primaries. $10.0 \% \mathrm{He}$ events fall in the 1st row (heavy) and $17.2 \%$ events fall in the 1st column (light). $20.4 \%$ He events fall in the 1st row (heavy) and $7.8 \%$ events fall in the 1 st column (light).

\section{Summary and Outlook}

We have explored a new method for determining the composition of inclined air showers on an event-by-event basis. This utlilizes the discrimination of muon-like signals in the air shower and using it to draw probability-density distributions for both proton and iron showers. Based on this, we classify events as light or heavy by assigning them marginal cumulative distribution values and marginal inverse cumulative distribution values. A verification of the procedure reveals that the method is effective. Further tests on other hadronic interaction models will be needed to determine the model-dependent performance of the analysis. With that, we can use the analysis method to determine the mass composition of inclined air showers measured with IceTop, and thereby provide an independent check to the composition measurement of quasi-vertical showers from the same detector array.

\section{References}

[1] IceCube Collaboration, R. Abbasi et al. Nucl. Instr. and Meth. in Phys. Res. A 700 (Feb., 2013) 188-220.

[2] IceCube Collaboration PoS ICRC2021 (these proceedings) 342.

[3] IceCube Collaboration, M. G. Aartsen et al. Phys. Rev. D 100 no. 8, (2019) 082002.

[4] IceCube Collaboration PoS ICRC2021 (these proceedings) 312.

[5] IceCube Collaboration PoS ICRC2021 (these proceedings) 323.

[6] IceCube Collaboration $P o S$ ICRC2021 (these proceedings) 357.

[7] A. Balagopal V., DOI: 10.5445/IR/1000091377. PhD thesis, 2019.

[8] D. Heck, J. Knapp, J. N. Capdevielle, G. Schatz, and T. Thouw. (1998) FZKA-6019.

[9] E.-J. Ahn et al. Phys. Rev. D 80 (Nov, 2009) 094003.

[10] E. M. Holt, F. G. Schröder, and A. Haungs Eur. Phys. J. C 79 no. 5, (2019) 371. 


\section{Full Author List: IceCube Collaboration}

R. Abbasi ${ }^{17}$, M. Ackermann ${ }^{59}$, J. Adams ${ }^{18}$, J. A. Aguilar ${ }^{12}$, M. Ahlers ${ }^{22}$, M. Ahrens ${ }^{50}$, C. Alispach ${ }^{28}$, A. A. Alves Jr. ${ }^{31}$, N. M. $\mathrm{Amin}^{42}$, R. An ${ }^{14}$, K. Andeen ${ }^{40}$, T. Anderson ${ }^{56}$, G. Anton ${ }^{26}$, C. Argüelles ${ }^{14}$, Y. Ashida ${ }^{38}$, S. Axani ${ }^{15}$, X. Bai ${ }^{46}$, A. Balagopal V. ${ }^{38}$, A. Barbano ${ }^{28}$, S. W. Barwick ${ }^{30}$, B. Bastian ${ }^{59}$, V. Basu ${ }^{38}$, S. Baur ${ }^{12}$, R. Bay ${ }^{8}$, J. J. Beatty ${ }^{20}, 21$, K.-H. Becker ${ }^{58}$, J. Becker Tjus ${ }^{11}$, C. Bellenghi $^{27}$, S. BenZvi ${ }^{48}$, D. Berley ${ }^{19}$, E. Bernardini ${ }^{59,60}$, D. Z. Besson ${ }^{34,61}$, G. Binder ${ }^{8,9}$, D. Bindig ${ }^{58}$, E. Blaufuss ${ }^{19}$, S. Blot ${ }^{59}$, M. Boddenberg ${ }^{1}$, F. Bontempo ${ }^{31}$, J. Borowka ${ }^{1}$, S. Böser ${ }^{39}$, O. Botner ${ }^{57}$, J. Böttcher ${ }^{1}$, E. Bourbeau ${ }^{22}$, F. Bradascio ${ }^{59}$, J. Braun ${ }^{38}$, S. Bron $^{28}$, J. Brostean-Kaiser ${ }^{59}$, S. Browne ${ }^{32}$, A. Burgman ${ }^{57}$, R. T. Burley ${ }^{2}$, R. S. Busse ${ }^{41}$, M. A. Campana ${ }^{45}$, E. G. Carnie-Bronca ${ }^{2}$, C. Chen ${ }^{6}$, D. Chirkin ${ }^{38}$, K. Choi ${ }^{52}$, B. A. Clark ${ }^{24}$, K. Clark ${ }^{33}$, L. Classen ${ }^{41}$, A. Colemann ${ }^{42}$, G. H. Collin ${ }^{15}$, J. M. Conrad ${ }^{15}$, P. Coppin $^{13}$, P. Correa ${ }^{13}$, D. F. Cowen ${ }^{55,56}$, R. Cross $^{48}$, C. Dappen ${ }^{1}$, P. Dave ${ }^{6}$, C. De Clercq ${ }^{13}$, J. J. DeLaunay ${ }^{56}$, H. Dembinski ${ }^{42}$, K. Deoskar $^{50}$, S. De Ridder ${ }^{29}$, A. Desai ${ }^{38}$, P. Desiati ${ }^{38}$, K. D. de Vries ${ }^{13}$, G. de Wasseige ${ }^{13}$, M. de With ${ }^{10}$, T. DeYoung ${ }^{24}$, S. Dharani ${ }^{1}$, A. Diaz ${ }^{15}$, J. C. Díaz-Vélez ${ }^{38}$, M. Dittmer ${ }^{41}$, H. Dujmovic ${ }^{31}$, M. Dunkman ${ }^{56}$, M. A. DuVernois ${ }^{38}$, E. Dvorak ${ }^{46}$, T. Ehrhardt ${ }^{39}$, P. Eller $^{27}$, R. Engel ${ }^{31,32}$, H. Erpenbeck ${ }^{1}$, J. Evans ${ }^{19}$, P. A. Evenson ${ }^{42}$, K. L. Fan ${ }^{19}$, A. R. Fazely ${ }^{7}$, S. Fiedlschuster ${ }^{26}$, A. T. Fienberg ${ }^{56}$, K. Filimonov ${ }^{8}$, C. Finley ${ }^{50}$, L. Fischer ${ }^{59}$, D. Fox ${ }^{55}$, A. Franckowiak ${ }^{11,59}$, E. Friedman ${ }^{19}$, A. Fritz ${ }^{39}$, P. Fürst ${ }^{1}$, T. K. Gaisser ${ }^{42}$, J. Gallagher $^{37}$, E. Ganster ${ }^{1}$, A. Garcia ${ }^{14}$, S. Garrappa ${ }^{59}$, L. Gerhardt ${ }^{9}$, A. Ghadimi ${ }^{54}$, C. Glaser ${ }^{57}$, T. Glauch ${ }^{27}$, T. Glüsenkamp ${ }^{26}$, A. Goldschmidt $^{9}$, J. G. Gonzalez ${ }^{42}$, S. Goswami ${ }^{54}$, D. Grant ${ }^{24}$, T. Grégoire ${ }^{56}$, S. Griswold ${ }^{48}$, M. Gündüz ${ }^{11}$, C. Günther ${ }^{1}$, C. Haack ${ }^{27}$,

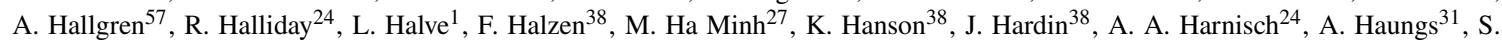
Hauser $^{1}$, D. Hebecker ${ }^{10}$, K. Helbing ${ }^{58}$, F. Henningsen ${ }^{27}$, E. C. Hettinger ${ }^{24}$, S. Hickford ${ }^{58}$, J. Hignight ${ }^{25}$, C. Hill ${ }^{16}$, G. C. Hill ${ }^{2}$, K. D.

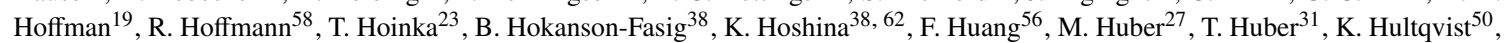
M. Hünnefeld ${ }^{23}$, R. Hussain ${ }^{38}$, S. In ${ }^{52}$, N. Iovine ${ }^{12}$, A. Ishihara ${ }^{16}$, M. Jansson ${ }^{50}$, G. S. Japaridze ${ }^{5}$, M. Jeong ${ }^{52}$, B. J. P. Jones ${ }^{4}$, D. Kang ${ }^{31}$, W. Kang ${ }^{52}$, X. Kang ${ }^{45}$, A. Kappes ${ }^{41}$, D. Kappesser ${ }^{39}$, T. Karg ${ }^{59}$, M. Karl ${ }^{27}$, A. Karle ${ }^{38}$, U. Katz ${ }^{26}$, M. Kauer ${ }^{38}$, M. Kellermann ${ }^{1}$, J. L. Kelley $^{38}$, A. Kheirandish ${ }^{56}$, K. Kin ${ }^{16}$, T. Kintscher ${ }^{59}$, J. Kiryluk ${ }^{51}$, S. R. Klein ${ }^{8,9}$, R. Koirala ${ }^{42}$, H. Kolanoski ${ }^{10}$, T. Kontrimas ${ }^{27}$, L. Köpke $^{39}$, C. Kopper ${ }^{24}$, S. Kopper ${ }^{54}$, D. J. Koskinen ${ }^{22}$, P. Koundal ${ }^{31}$, M. Kovacevich ${ }^{45}$, M. Kowalski ${ }^{10,59}$, T. Kozynets ${ }^{22}$, E. Kun ${ }^{11}$, N. Kurahashi ${ }^{45}$, N. Lad ${ }^{59}$, C. Lagunas Gualda ${ }^{59}$, J. L. Lanfranchi ${ }^{56}$, M. J. Larson ${ }^{19}$, F. Lauber ${ }^{58}$, J. P. Lazar ${ }^{14,38}$, J. W. Lee ${ }^{52}$, K. Leonard $^{38}$, A. Leszczyńska ${ }^{32}$, Y. Li ${ }^{56}$, M. Lincetto ${ }^{11}$, Q. R. Liu ${ }^{38}$, M. Liubarska ${ }^{25}$, E. Lohfink ${ }^{39}$, C. J. Lozano Mariscal ${ }^{41}$, L. Lu ${ }^{38}$,

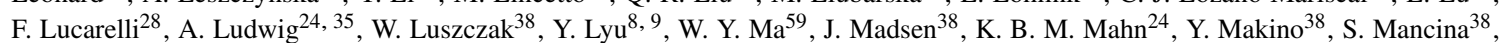

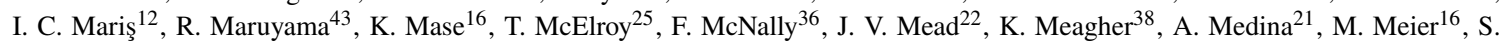
Meighen-Berger $^{27}$, J. Micallef ${ }^{24}$, D. Mockler ${ }^{12}$, T. Montaruli ${ }^{28}$, R. W. Moore ${ }^{25}$, R. Morse ${ }^{38}$, M. Moulai ${ }^{15}$, R. Naab ${ }^{59}$, R. Nagai ${ }^{16}$, U. Naumann $^{58}$, J. Necker ${ }^{59}$, L. V. Nguyễn ${ }^{24}$, H. Niederhausen ${ }^{27}$, M. U. Nisa ${ }^{24}$, S. C. Nowicki ${ }^{24}$, D. R. Nygren ${ }^{9}$, A. Obertacke Pollmann ${ }^{58}$, M. Oehler ${ }^{31}$, A. Olivas ${ }^{19}$, E. O'Sullivan ${ }^{57}$, H. Pandya ${ }^{42}$, D. V. Pankova ${ }^{56}$, N. Park ${ }^{33}$, G. K. Parker ${ }^{4}$, E. N. Paudel ${ }^{42}$, L. Paul ${ }^{40}$, C. Pérez de los $\operatorname{Heros}^{57}$, L. Peters ${ }^{1}$, J. Peterson ${ }^{38}$, S. Philippen ${ }^{1}$, D. Pieloth ${ }^{23}$, S. Pieper ${ }^{58}$, M. Pittermann ${ }^{32}$, A. Pizzuto ${ }^{38}$, M. Plum ${ }^{40}$, Y. Popovych $^{39}$, A. Porcelli ${ }^{29}$, M. Prado Rodriguez ${ }^{38}$, P. B. Price ${ }^{8}$, B. Pries ${ }^{24}$, G. T. Przybylski ${ }^{9}$, C. Raab ${ }^{12}$, A. Raissi ${ }^{18}$, M. Rameez ${ }^{22}$, K. $^{2}$. Rawlins $^{3}$, I. C. Rea ${ }^{27}$, A. Rehman ${ }^{42}$, P. Reichherzer ${ }^{11}$, R. Reimann ${ }^{1}$, G. Renzi ${ }^{12}$, E. Resconi ${ }^{27}$, S. Reusch ${ }^{59}$, W. Rhode ${ }^{23}$, M. Richman $^{45}$, B. Riedel ${ }^{38}$, E. J. Roberts ${ }^{2}$, S. Robertson ${ }^{8,9}$, G. Roellinghoff ${ }^{52}$, M. Rongen ${ }^{39}$, C. Rott ${ }^{49}, 52$, T. Ruhe ${ }^{23}$, D. Ryckbosch ${ }^{29}$, D. Rysewyk $\mathrm{Cantu}^{24}$, I. Safa ${ }^{14,38}$, J. Saffer ${ }^{32}$, S. E. Sanchez Herrera ${ }^{24}$, A. Sandrock ${ }^{23}$, J. Sandroos ${ }^{39}$, M. Santander ${ }^{54}$, S. Sarkar ${ }^{44}$, S. Sarkar ${ }^{25}$, K. Satalecka $^{59}$, M. Scharf ${ }^{1}$, M. Schaufel ${ }^{1}$, H. Schieler ${ }^{31}$, S. Schindler ${ }^{26}$, P. Schlunder ${ }^{23}$, T. Schmidt ${ }^{19}$, A. Schneider ${ }^{38}$, J. Schneider ${ }^{26}$, F. G. Schröder ${ }^{31,42}$, L. Schumacher ${ }^{27}$, G. Schwefer ${ }^{1}$, S. Sclafani ${ }^{45}$, D. Seckel ${ }^{42}$, S. Seunarine ${ }^{47}$, A. Sharma ${ }^{57}$, S. Shefali ${ }^{32}$, M. Silva $^{38}$, B. Skrzypek ${ }^{14}$, B. Smithers ${ }^{4}$, R. Snihur ${ }^{38}$, J. Soedingrekso ${ }^{23}$, D. $\operatorname{Soldin}^{42}$, C. Spannfellner ${ }^{27}$, G. M. Spiczak ${ }^{47}$, C. Spiering ${ }^{59,61}$, J. Stachurska $^{59}$, M. Stamatikos ${ }^{21}$, T. Stanev ${ }^{42}$, R. Stein ${ }^{59}$, J. Stettner ${ }^{1}$, A. Steuer ${ }^{39}$, T. Stezelberger ${ }^{9}$, T. Stürwald ${ }^{58}$, T. Stuttard ${ }^{22}$, G. W. Sullivan $^{19}$, I. Taboada ${ }^{6}$, F. Tenholt ${ }^{11}$, S. Ter-Antonyan ${ }^{7}$, S. Tilav ${ }^{42}$, F. Tischbein ${ }^{1}$, K. Tollefson $^{24}$, L. Tomankova ${ }^{11}$, C. Tönnis ${ }^{53}$, S.

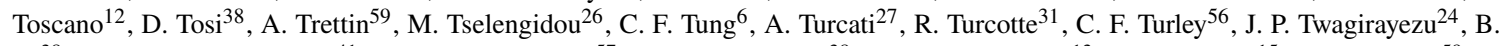
$\mathrm{Ty}^{38}$, M. A. Unland Elorrieta ${ }^{41}$, N. Valtonen-Mattila ${ }^{57}$, J. Vandenbroucke ${ }^{38}$, N. van Eijndhoven ${ }^{13}$, D. Vannerom ${ }^{15}$, J. van Santen ${ }^{59}, \mathrm{~S}$. Verpoest $^{29}$, M. Vraeghe ${ }^{29}$, C. Walck ${ }^{50}$, T. B. Watson ${ }^{4}$, C. Weaver ${ }^{24}$, P. Weigel ${ }^{15}$, A. Weindl ${ }^{31}$, M. J. Weiss ${ }^{56}$, J. Weldert ${ }^{39}$, C. Wendt $^{38}$, J. Werthebach ${ }^{23}$, M. Weyrauch ${ }^{32}$, N. Whitehorn ${ }^{24,35}$, C. H. Wiebusch ${ }^{1}$, D. R. Williams ${ }^{54}$, M. Wolf ${ }^{27}$, K. Woschnagg ${ }^{8}$, G. Wrede ${ }^{26}$, J. Wulff ${ }^{11}$, X. W. Xu $u^{7}$, Y. Xu ${ }^{51}$, J. P. Yanez ${ }^{25}$, S. Yoshida ${ }^{16}$, S. Yu ${ }^{24}$, T. Yuan ${ }^{38}$, Z. Zhang ${ }^{51}$

${ }^{1}$ III. Physikalisches Institut, RWTH Aachen University, D-52056 Aachen, Germany

2 Department of Physics, University of Adelaide, Adelaide, 5005, Australia

${ }^{3}$ Dept. of Physics and Astronomy, University of Alaska Anchorage, 3211 Providence Dr., Anchorage, AK 99508, USA

${ }^{4}$ Dept. of Physics, University of Texas at Arlington, 502 Yates St., Science Hall Rm 108, Box 19059, Arlington, TX 76019, USA

5 CTSPS, Clark-Atlanta University, Atlanta, GA 30314, USA

${ }^{6}$ School of Physics and Center for Relativistic Astrophysics, Georgia Institute of Technology, Atlanta, GA 30332, USA

${ }^{7}$ Dept. of Physics, Southern University, Baton Rouge, LA 70813, USA

${ }^{8}$ Dept. of Physics, University of California, Berkeley, CA 94720, USA

${ }^{9}$ Lawrence Berkeley National Laboratory, Berkeley, CA 94720, USA

${ }^{10}$ Institut für Physik, Humboldt-Universität zu Berlin, D-12489 Berlin, Germany

${ }^{11}$ Fakultät für Physik \& Astronomie, Ruhr-Universität Bochum, D-44780 Bochum, Germany

12 Université Libre de Bruxelles, Science Faculty CP230, B-1050 Brussels, Belgium

13 Vrije Universiteit Brussel (VUB), Dienst ELEM, B-1050 Brussels, Belgium

14 Department of Physics and Laboratory for Particle Physics and Cosmology, Harvard University, Cambridge, MA 02138, USA

15 Dept. of Physics, Massachusetts Institute of Technology, Cambridge, MA 02139, USA 
${ }^{16}$ Dept. of Physics and Institute for Global Prominent Research, Chiba University, Chiba 263-8522, Japan

${ }^{17}$ Department of Physics, Loyola University Chicago, Chicago, IL 60660, USA

${ }^{18}$ Dept. of Physics and Astronomy, University of Canterbury, Private Bag 4800, Christchurch, New Zealand

${ }^{19}$ Dept. of Physics, University of Maryland, College Park, MD 20742, USA

${ }^{20}$ Dept. of Astronomy, Ohio State University, Columbus, OH 43210, USA

${ }^{21}$ Dept. of Physics and Center for Cosmology and Astro-Particle Physics, Ohio State University, Columbus, OH 43210, USA

${ }^{22}$ Niels Bohr Institute, University of Copenhagen, DK-2100 Copenhagen, Denmark

${ }^{23}$ Dept. of Physics, TU Dortmund University, D-44221 Dortmund, Germany

24 Dept. of Physics and Astronomy, Michigan State University, East Lansing, MI 48824, USA

${ }^{25}$ Dept. of Physics, University of Alberta, Edmonton, Alberta, Canada T6G 2E1

${ }^{26}$ Erlangen Centre for Astroparticle Physics, Friedrich-Alexander-Universität Erlangen-Nürnberg, D-91058 Erlangen, Germany

${ }^{27}$ Physik-department, Technische Universität München, D-85748 Garching, Germany

${ }^{28}$ Département de physique nucléaire et corpusculaire, Université de Genève, CH-1211 Genève, Switzerland

${ }^{29}$ Dept. of Physics and Astronomy, University of Gent, B-9000 Gent, Belgium

${ }^{30}$ Dept. of Physics and Astronomy, University of California, Irvine, CA 92697, USA

${ }^{31}$ Karlsruhe Institute of Technology, Institute for Astroparticle Physics, D-76021 Karlsruhe, Germany

${ }^{32}$ Karlsruhe Institute of Technology, Institute of Experimental Particle Physics, D-76021 Karlsruhe, Germany

${ }^{3}$ Dept. of Physics, Engineering Physics, and Astronomy, Queen's University, Kingston, ON K7L 3N6, Canada

${ }^{34}$ Dept. of Physics and Astronomy, University of Kansas, Lawrence, KS 66045, USA

${ }^{35}$ Department of Physics and Astronomy, UCLA, Los Angeles, CA 90095, USA

${ }^{36}$ Department of Physics, Mercer University, Macon, GA 31207-0001, USA

${ }^{37}$ Dept. of Astronomy, University of Wisconsin-Madison, Madison, WI 53706, USA

${ }^{38}$ Dept. of Physics and Wisconsin IceCube Particle Astrophysics Center, University of Wisconsin-Madison, Madison, WI 53706, USA

${ }^{39}$ Institute of Physics, University of Mainz, Staudinger Weg 7, D-55099 Mainz, Germany

${ }^{40}$ Department of Physics, Marquette University, Milwaukee, WI, 53201, USA

${ }^{41}$ Institut für Kernphysik, Westfälische Wilhelms-Universität Münster, D-48149 Münster, Germany

42 Bartol Research Institute and Dept. of Physics and Astronomy, University of Delaware, Newark, DE 19716, USA

${ }^{43}$ Dept. of Physics, Yale University, New Haven, CT 06520, USA

44 Dept. of Physics, University of Oxford, Parks Road, Oxford OX1 3PU, UK

${ }^{45}$ Dept. of Physics, Drexel University, 3141 Chestnut Street, Philadelphia, PA 19104, USA

${ }^{46}$ Physics Department, South Dakota School of Mines and Technology, Rapid City, SD 57701, USA

${ }^{47}$ Dept. of Physics, University of Wisconsin, River Falls, WI 54022, USA

${ }^{48}$ Dept. of Physics and Astronomy, University of Rochester, Rochester, NY 14627, USA

${ }^{49}$ Department of Physics and Astronomy, University of Utah, Salt Lake City, UT 84112, USA

${ }^{50}$ Oskar Klein Centre and Dept. of Physics, Stockholm University, SE-10691 Stockholm, Sweden

${ }^{51}$ Dept. of Physics and Astronomy, Stony Brook University, Stony Brook, NY 11794-3800, USA

52 Dept. of Physics, Sungkyunkwan University, Suwon 16419, Korea

${ }^{53}$ Institute of Basic Science, Sungkyunkwan University, Suwon 16419, Korea

${ }^{54}$ Dept. of Physics and Astronomy, University of Alabama, Tuscaloosa, AL 35487, USA

${ }^{55}$ Dept. of Astronomy and Astrophysics, Pennsylvania State University, University Park, PA 16802, USA

${ }^{56}$ Dept. of Physics, Pennsylvania State University, University Park, PA 16802, USA

57 Dept. of Physics and Astronomy, Uppsala University, Box 516, S-75120 Uppsala, Sweden

${ }^{58}$ Dept. of Physics, University of Wuppertal, D-42119 Wuppertal, Germany

${ }^{59}$ DESY, D-15738 Zeuthen, Germany

${ }^{60}$ Università di Padova, I-35131 Padova, Italy

${ }^{61}$ National Research Nuclear University, Moscow Engineering Physics Institute (MEPhI), Moscow 115409, Russia

${ }^{62}$ Earthquake Research Institute, University of Tokyo, Bunkyo, Tokyo 113-0032, Japan

\section{Acknowledgements}

USA - U.S. National Science Foundation-Office of Polar Programs, U.S. National Science Foundation-Physics Division, U.S. National Science Foundation-EPSCoR, Wisconsin Alumni Research Foundation, Center for High Throughput Computing (CHTC) at the University of Wisconsin-Madison, Open Science Grid (OSG), Extreme Science and Engineering Discovery Environment (XSEDE), Frontera computing project at the Texas Advanced Computing Center, U.S. Department of Energy-National Energy Research Scientific Computing Center, Particle astrophysics research computing center at the University of Maryland, Institute for Cyber-Enabled Research at Michigan State University, and Astroparticle physics computational facility at Marquette University; Belgium - Funds for Scientific Research (FRS-FNRS and FWO), FWO Odysseus and Big Science programmes, and Belgian Federal Science Policy Office (Belspo); Germany - Bundesministerium für Bildung und Forschung (BMBF), Deutsche Forschungsgemeinschaft (DFG), Helmholtz Alliance for Astroparticle Physics (HAP), Initiative and Networking Fund of the Helmholtz Association, Deutsches Elektronen Synchrotron (DESY), and High Performance Computing cluster of the RWTH Aachen; Sweden - Swedish Research Council, Swedish Polar Research Secretariat, Swedish National Infrastructure for Computing (SNIC), and Knut and Alice Wallenberg Foundation; Australia - Australian 
Research Council; Canada - Natural Sciences and Engineering Research Council of Canada, Calcul Québec, Compute Ontario, Canada Foundation for Innovation, WestGrid, and Compute Canada; Denmark - Villum Fonden and Carlsberg Foundation; New Zealand Marsden Fund; Japan - Japan Society for Promotion of Science (JSPS) and Institute for Global Prominent Research (IGPR) of Chiba University; Korea - National Research Foundation of Korea (NRF); Switzerland - Swiss National Science Foundation (SNSF); United Kingdom - Department of Physics, University of Oxford. 